\title{
Distinctive features of persuasion and deliberation dialogues
}

\author{
Katie Atkinson $^{\mathrm{a} *}$, Trevor Bench-Capon ${ }^{\mathrm{a}}$ and Douglas Walton ${ }^{\mathrm{b}}$ \\ ${ }^{a}$ Department of Computer Science, University of Liverpool, L69 3BX, England, UK; ${ }^{b}$ CRRAR, University \\ of Windsor, Windsor, Ontario, Canada
}

(Received 22 December 2011; final version received 10 June 2012)

\begin{abstract}
The distinction between action persuasion dialogues and deliberation dialogues is not always obvious at first sight. In this paper, we provide a characterisation of both types of dialogues that draws out the distinctive features of each. It is important to recognise the distinctions since participants in both types of dialogues will have different aims, which in turn affects whether a successful outcome can be reached. Such dialogues are typically conducted by exchanging arguments for and against certain options. The moves of the dialogue are designed to facilitate such exchanges. In particular, we show how the pre- and post-conditions for the use of particular moves in the dialogues are very different depending upon whether they are used as part of a persuasion over action or a deliberation dialogue. We draw out the distinctions with reference to a running example that we also present as a logic program in order to give a clear characterisation of the two types of dialogues, which is intended to enable them to be used more effectively within systems requiring automated communication.
\end{abstract}

Keywords: deliberation; persuasion over action; dialogue

\section{Introduction}

The influential dialogue typology of Walton and Krabbe (1995) has been used as a basis for developing protocols for a variety of different types of dialogues for use in agent systems. For example, early work in agent communication such as Reed (1998) recognised the need to differentiate between different dialogue types to facilitate the design of communication protocols for complex multi-agent societies. Other work such as Amgoud, Maudet, and Parsons (2002) began to explicitly develop argumentation-based semantics for agent communication languages. Argumentation-based dialogues are discussed in general in Reed and Norman (2003), but other work has focussed on specifying the interactions for specific dialogue types. For example, see Black and Hunter (2009) for inquiry dialogues; Rahwan et al. (2003) and McBurney, van Eijk, Parsons, and Amgoud (2003) for negotiation; Prakken (2006) and Dignum, Dunin-Keplicz, and Verbrugge (2001) for persuasion dialogues; and McBurney, Hitchcock, and Parsons (2007) and Dunin-Keplicz, Strachocka, and Verbrugge (2011) for deliberations. In this paper, we will look at the last two of these, both in a particular context.

In Walton and Krabbe (1995), persuasion was characterised as a dialogue that involves an attempt by one participant to have another participant endorse some proposition or statement. The statement at issue may concern the beliefs of the participants or proposals for action, and the dialogue may or may not involve conflict between the participants. Deliberation is characterised as a dialogue occurring when two or more parties attempt to agree on an action, or a course of action, in some situation. One common situation in which persuasion and deliberation occur is when a group of agents must collectively choose between several options, each of which has several

*Corresponding author. Email: K.M.Atkinson@liverpool.ac.uk, K.M.Atkinson@csc.liv.ac.uk 
features to which different agents will ascribe different degrees of importance. When buying a car, one person will be most interested in safety, another in speed, another in comfort. For cameras, some will require the best quality, whereas others will value compactness or price more highly. A familiar example for academic scientists is where a group of colleagues must decide on a restaurant at a conference, and we will use this as an illustration throughout this paper. In such a situation, we may find either persuasion or deliberation or even shifts between the two. We will examine these dialogues so as to make clear the distinction between persuasion and deliberation, which has often become rather blurred in the computational argumentation literature. Even though different views have been taken on deliberation, such as in Tang and Parsons (2005), McBurney et al. (2007) and Black and Atkinson (2009), it is not always made entirely clear where deliberation ends and persuasion starts in matters concerning reasoning about what to do.

There is a pervasive problem in argumentation studies that there seems to be no firm and precise basis for distinguishing, even in the most common kinds of argumentative discussions that are often cited as the leading examples of instances of rational argumentation, whether the discussion should be classified as a persuasion dialogue and a deliberation dialogue. The pervasiveness of the problem can be indicated by listing the topics of some recently featured debate digest articles taken from Debatepedia: ${ }^{1}$

- Should there be a ban on sales of violent video games to minors?

- Should there be mandatory ultrasounds before abortions?

- Should colleges ban fraternities?

- Should public schools be allowed to teach creationism alongside evolution as part of their science curriculum?

- Should there be a worldwide ban on the use of driftnets to catch fish, including within each country's Exclusive Economic Zone?

- Should governments legalise all drugs?

- Should illegal immigrants in the United States be allowed to obtain drivers' licenses?

- Should the Roman Catholic Church change its current position of forbidding the use of contraception?

In each case, the topic of the debate concerns the decision to take action. For example, in the first item on the list, the decision is whether there should be a ban on sales of violent video games to minors. The second item concerns the decision on whether there should be mandatory ultrasounds before abortions. These observations about each debate being about a decision to take action of a certain kind suggest, at least on an initial superficial impression, that the debate should be structured in the format of a deliberation dialogue.

However, what needs to be noticed is that in each case, the debaters who are discussing the issue are not in a position to actually make the decision whether to take the action or not, based on their discussion of it. For example, the debaters who discussed the issue on the Debatepedia website are not in a position to take action to ban fraternities in all colleges, even though the debate may have come to the conclusion that all colleges should ban fraternities. Hence, we can conclude that it would be improper to classify each of these debates as deliberations. They should really be classified as persuasion dialogues over action. Looking at the list given above as a sample of many of the other featured debates on Debatepedia, the hypothesis is suggested that many of the most common examples of argumentation treated in the argumentation literature fall into this category.

In this paper, we will take it that the persuasion or deliberation is about some action that should be done. There has been some disagreement as to whether persuasion can be over actions. Walton (1998) seems to suggest not. However, in a later book (Walton 2006), we find the following quotation (p. 174): "[...] such opposition in a persuasion dialogue, whether it be a dispute or dissent, can represent a factual disagreement or a disagreement about whether some action or kind of action is a good policy or not". The example cited (on p. 174) is a dispute about tipping, representing a disagreement on whether a kind of action, mainly tipping, is generally a good policy 
or not. Walton classifies this kind of case as a persuasion dialogue, "even though it is about actions and about whether some specific course of action or policy for action is good or not" (Walton, pp. 174-175). Other work from the agents literature, such as Dignum et al. (2001), also addresses the point that persuasion can involve convincing other agents to do something or to commit to an intention.

Moreover, it is clear that we are, in ordinary language, fully prepared to speak of persuading someone to do something. Rather than a distinction between actions and propositions, we believe that the correct distinction is related to directions of fit, a distinction made by Searle (2003). Searle distinguishes theoretical reasoning, reasoning about what is the case, from practical reasoning, reasoning about what it is desired to be the case and what should be done to realise those desires. In the first case, it is necessary to fit one's beliefs to the world, whereas in the second, the idea is to make the world fit one's desires, in so far as one has the capacity to do so. In these terms, inquiry represents an attempt to better fit the beliefs of the agents to the world, and deliberation how best to make the world fit the collective desires of the agents. Persuasion can be about either. Note, however, that when we have several participating agents, we have several, probably different, sets of desires to consider. In deliberation, no set of desires should be given pre-eminence, but, as discussed in Bench-Capon (2002), in persuasion, it is the desires of an agent being persuaded that matter: an agent being persuaded is fully entitled to use its own preferences to assess any proposition or proposal and need not consider the desires and preferences of the persuading agent at all. The construction of collective preferences rather than the use of those of a particular individual is a key feature of deliberation and lies at the heart of the differences between the two dialogue types.

From the typology in Walton and Krabbe (1995), we learn that dialogue types are characterised by three aspects: an initial situation, a collective goal and the individual goals of the participants. The third element may give rise to an asymmetry between the participants where they have different individual goals. For persuasion, the initial situation is a conflict: the agents do not agree as to the best option. In deliberation, the initial situation is one of uncertainty: while the individuals may have opinions about what the best option for themselves is, they do not know which option is collectively acceptable, the best for the group as a whole. In persuasion and deliberation alike, the collective goal is to come to an agreement as to the best option, but what counts as best differs. In persuasion, the best option is the one that is best for the agent being persuaded; in deliberation, the best option is the one that is best for the group considered as a whole. With regard to the individual goals, persuasion is asymmetric: the persuader wishes to convince the other, whereas the persuadee, if cooperative, wishes to explore the possibility that its currently preferred option is not in fact best for it in the light of information known to the persuader and, if uncooperative, may wish to defend its own option or even convert the persuader. Deliberation is symmetric: all the agents involved wish to determine which option is the best for them considered as a group.

Although these aspects of the dialogue types are quite different, it may be unclear as to which dialogue type is in progress, since the performatives used in both persuasion and deliberation can be the same, and there is no explicit statement of the goals at the outset of the dialogues to make it clear what kind of dialogue is to follow. Thus, the two types can appear the same to an observer, and the agents participating may even differ as to the type of dialogue they believe they are engaged in. It is, however, important for both automated systems and humans to be aware of (and agree on) the dialogue type in which they are participating if they are to communicate effectively. Indeed, this is one of the desiderata of argumentation protocols set out in McBurney, Parsons, and Wooldridge (2002). The reason that this is so important is that the pragmatic meanings of the various performatives differ between the dialogue types. It is these differences in the pragmatic meanings supplied by the dialogue contexts that we especially wish 
to explore in this paper, as it is here that the real distinction, and the distinction that matters, can be found.

The contribution of the paper is threefold. First, we provide an analysis of dialogues involving arguments about action that clearly differentiates persuasion from deliberation. Second, from this analysis, we make these differences precise by explicitly identifying the pragmatic forces of the speech acts used in the different dialogue types. Third, we express these differences as additional pre- and post-conditions on the speech acts used in Prakken (2006). This analysis provides a principled basis for the implementation of persuasion and deliberation dialogues that can avoid conflation of the two types.

The starting point for our analysis will be Prakken (2006) account of persuasion dialogues. In particular, we will adopt his dialogue moves as the basis of the speech acts we will use. Each of these speech acts will be specified in terms of its pre- and post-conditions, and we will see that it is with respect to these conditions that the characteristic differences between persuasion and deliberation manifest themselves. We will first, however, present motivating examples of persuasion and deliberation dialogues. ${ }^{2}$ These dialogues will form a running example that we will use to illustrate our points throughout the paper. The scenarios for persuasion and deliberation will be presented informally in Section 2 and made concrete as logic programs in Section 3. Section 4 will analyse the contrasts between the two dialogue types revealed in the scenarios. In Section 5, these contrasts will be expressed in terms of additional pre- and post-conditions on the speech acts of Prakken (2006). Section 6 will discuss some issues arising that suggest directions for future work, and Section 7 offers some concluding remarks.

\section{Two scenarios}

We will consider here a scenario for persuasion and a scenario for deliberation. Both involve the choice of a restaurant, with a group of people, perhaps academics attending a conference in a strange city, needing to fix on a place to eat. In our scenarios, there will be three people: Jane, Harry and George. There are three options: La Zingara, an Italian restaurant; the Thai Palace, a Thai restaurant; and Nosh, a fast food restaurant.

\subsection{Scenario 1: persuasion}

In the persuasion dialogue, initially Harry wants to go to the Thai Palace, Jane wants to go to La Zingara and George to Nosh.

P1. Jane: Where shall we eat?

P2. Harry: I propose going to the Thai Palace. It has Thai food.

P3. George: The Thai Palace is in the east end, and that is quite far away.

P4. Harry: I heard a colleague say that the food at the Thai Palace is very good.

P5. George: Nosh is popular with students, so it must be a very good restaurant.

P6. Harry: I don't think so. Students tend to go for low-budget fast food that is not very good.

P7. Jane: My husband and I have eaten at La Zingara. The food is really good. Italian food is also very healthy. It is a Mediterranean diet, with tomatoes, olive oil and fresh salad.

P8. Harry: Thai food is very healthy. It has lots of vegetables in it.

P9. Harry: The Thai Palace is cheaper than La Zingara.

P10. George: We would have to take a cab to get there and back. That would cost about ten dollars each.

P11. Harry: That would still be as cheap as La Zingara

P12. George: I'm persuaded.

P13. Jane: So am I. The Thai Palace it is. 


\subsection{Scenario 2: deliberation}

D1. Jane: Where shall we eat?

D2. Harry: There is the Thai Palace. It has Thai food.

D3. George: The Thai Palace is in the east end, and that is quite far away. Nosh is very close. It's right across the street.

D4. Jane: La Zingara is on Erie Street, which is fairly close. My husband and I have eaten at La Zingara. The food is really good.

D5. George: The prices at Nosh are cheaper than those at La Zingara.

D6. Harry: You can't get wine at Nosh. All of us want to have wine with dinner.

D7. Jane: Nosh lacks atmosphere. This is a special occasion. We want to go to a place with atmosphere.

D8. George: La Zingara has atmosphere.

D9. Harry: I heard a colleague say that the food at the Thai Palace is very good.

D10. George: None of us has a car. We would have to take a cab to get there and back. That would cost about ten dollars each.

D11. Harry: Thai food is very healthy. It has lots of vegetables in it.

D12. Jane: Italian food is also very healthy.

D13. George: We want a place with good food and atmosphere so it is either La Zingara or the Thai Palace. Both of them would fit the bill.

D14. Jane: We also want wine, but that doesn't help us choose.

D15. Harry: Let's make it La Zingara, since it's closer.

D16. George: Yes, I wouldn't want to add the cab fare to an expensive meal.

D17. Jane: Ok. I'm hungry so I'd like to eat sooner rather than later. Let's go to La Zingara.

As the dialogues proceed, agents become committed both to objective facts and to the subjective positions as to the criteria they will use to determine acceptability. The issue of commitment is motivated by Walton and Krabbe's (1995) original dialogue typology (as indicated by the title of their book). As such, in our analysis of the scenarios, the precise commitment agents enter into by using the various speech acts is of crucial importance in characterising the dialogue types, as we will show below.

\subsection{Commentary on the scenarios}

In the dialogues, arguments are put forward that take the form of options presented that should be accepted on the basis of having certain features. Whether they have these features is intended to be an objective matter, whereas whether these features make the option desirable is a subjective matter about which agents may rationally agree to disagree.

The two dialogues are very similar. They use the same speech acts, many of the arguments are the same in both dialogues and the underlying views of the three participants do not change between the dialogues. But there are some crucial differences.

In the persuasion dialogue, it is Harry who wants to go the Thai Palace, and so Harry advances all the pro arguments, while George and Jane make objections. Their objections indicate the points on which they wish to be convinced. George objects to the distance, which could be a matter of time or money. All of them want a good meal, and so George abandons Nosh when his reason for thinking the food is good there is rebutted. Jane is concerned that the food be healthy. George is persuaded on grounds of cost, and Jane accepts once her doubts about the healthiness of Thai cuisine have been met.

Whereas in the persuasion scenario, Harry is committed to making a case for the Thai Palace, in the deliberation scenario, no one is committed to any of the proposals, although they may have their preferences. In this dialogue, first several options emerge, neutrally, without any commitment on the part of the person introducing them, and then a number of criteria emerge together with how 
the options stand in relation to these criteria. While individual preferences may inform the options and criteria put forward by the various agents, they play no explicit role, unlike in persuasion where the preferences of the agent being persuaded must be satisfied if persuasion is to occur. In deliberation, some criteria will emerge as more important than others: above, wine and atmosphere are established as more important than cost. Finally, the options are assessed according to the criteria that have been chosen in the discussion, and the best fit is chosen by mutual agreement.

We will base our dialogues on the performatives given for persuasion dialogues in Prakken (2006). The form of these performatives carries with it the strong presupposition that the knowledge of the participants will be represented in the form of facts and rules (at least some of which are defeasible) and that the participants are able to reason with these. In order to make our example concrete, we will provide an example of the facts and rules sufficient to produce the dialogues in the above scenarios. We will therefore provide knowledge bases for our participants. We will use Prolog, as the most readily available logic programming language, to express these knowledge bases.

\section{Representation in Prolog}

\subsection{Example scenario}

Let us suppose that each agent $a g_{i}$ has an individual knowledge base $K B_{a g_{i}}$, comprising some facts $F_{a g_{i}}$ and some rules $R_{a g_{i}}$, which is expressed as a Prolog program:

$$
K B_{a g_{i}} \equiv R_{a g_{i}} \cup F_{a g_{i}} \equiv \Pi_{a g_{i}}
$$

These rules may relate to matters of fact (e.g. R4 below) or procedures (e.g. R7 below). Here, the individual KBs should differ only according to differences in the completeness of knowledge. Rules may also be heuristics, like R2 below, which some individuals may adopt and others may not adopt. Importantly, rules also express the preferences of an individual, such as HR1, GR1 and JR1 below. These preference rules will normally differ between individuals, as tastes and aspirations are expected to differ. Now consider a persuasion in which proponent $P$ is attempting to persuade some other agent opponent $O$, that some conclusion $c(a)$ is the case. Let $\operatorname{demo}(\Pi, c(a))$ be true if and only if $c(a)$ can be shown from $\Pi$. Now the initial position is

$\operatorname{demo}\left(\Pi_{P}, c(a)\right)$ and not demo $\left(\Pi_{O}, c(a)\right)$ and the desired result of the dialogue is that $\operatorname{demo}\left(\Pi_{O} \cup \Delta, c(a)\right)$ where $\Delta \subseteq \Pi_{P}$.

The dialogue is thus supposed to elicit a suitable set of facts and rules from $P$ to enable $O$ to derive $c(a)$ for some $a$.

In the persuasion scenario, the conclusion that Harry is trying to establish is acceptableRestaurant (thaiPalace) (which is an example of $c(a)$ ), on the basis of a number of facts describing the various restaurants. We can begin by representing the attributes of a restaurant. Let these be

- the distance to get to the restaurant (journey time in minutes, cost in dollars if cab necessary),

- the speed of service (time taken for a meal in minutes),

- the quality of the food (good, ok, poor),

- the price of a meal (dollars),

- whether the atmosphere is good (true or false),

- whether the restaurant is licensed to sell wine (true or false),

- the style of cuisine (Thai, Italian, American, etc.),

- whether the cuisine is healthy (true or false). 
Putting these into Prolog, we obtain, for the example scenarios: ${ }^{3}$

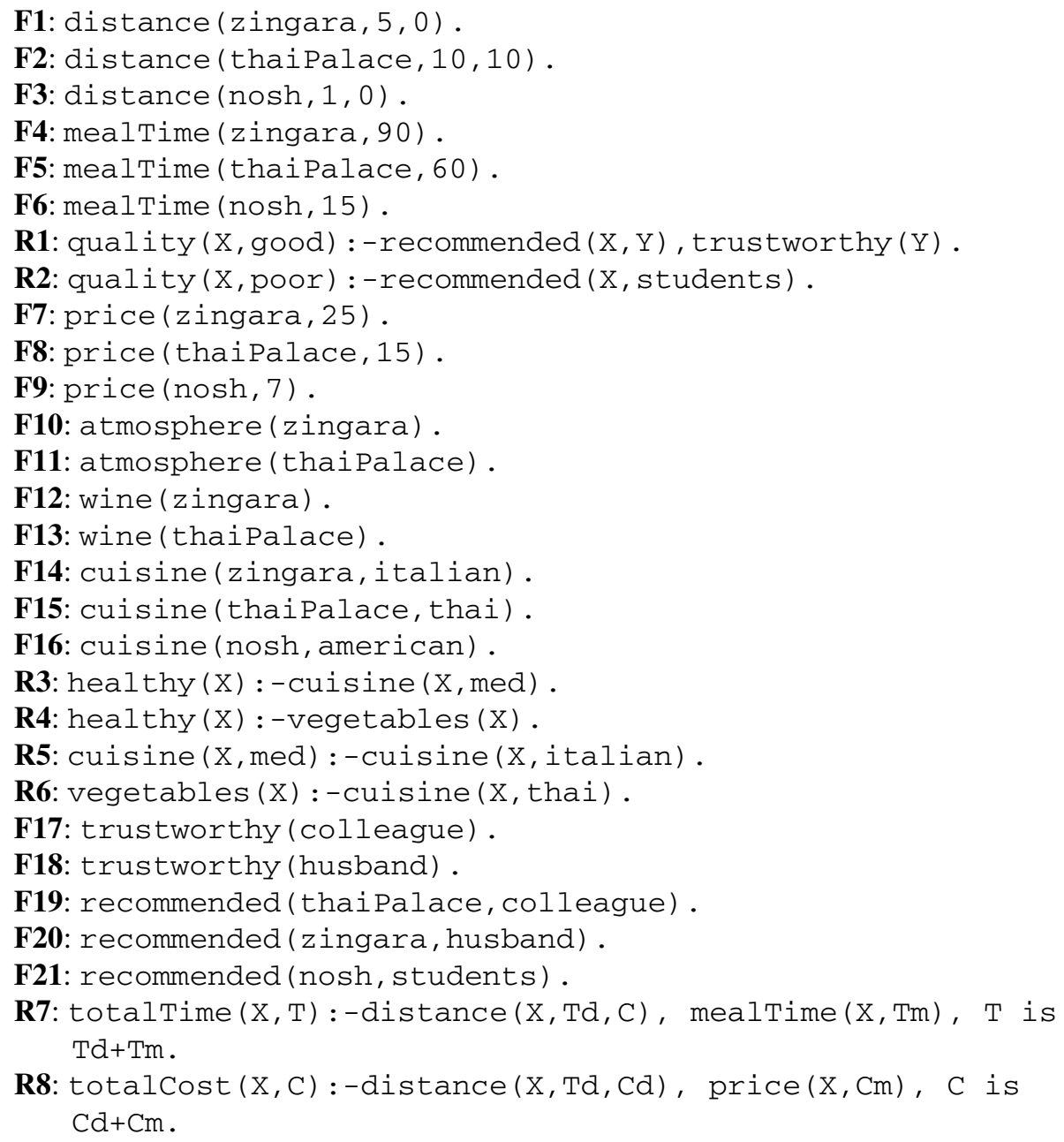

Each of the three participants will have his or her own, individual, notions as to what makes an acceptable restaurant. These are expressed as, for example, the following, where HR1 denotes Harry's rule, GR1 denotes George's rule and JR1 denotes Jane's rule:

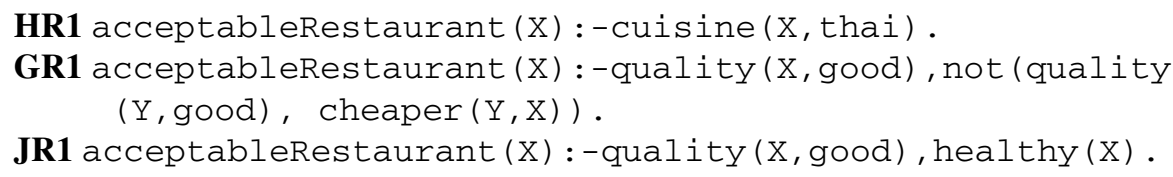

We also need an additional rule to define cheaper (where cheaper $(X, Y)$ stands for "Y is cheaper than $X$ ") and, for completeness, will also add one for quicker (where quicker $(X, Y)$ stands for "Y is quicker than $\mathrm{X}$ ").

R9: cheaper $(X, Y)$ : -totalCost $(X, C X)$, totalCost $(Y, C Y), C Y<C X$. R10: quicker $(\mathrm{X}, \mathrm{Y})$ :-totalTime $(\mathrm{X}, \mathrm{TX})$, totalTime $(\mathrm{Y}, \mathrm{TY}), \mathrm{TY}<\mathrm{TX}$. 
Thus, Harry desires only Thai food, George wants good food, but at the lowest cost possible, and Jane wants good food, but it must be healthy.

We can now use these facts and rules to construct the initial knowledge bases of Harry, Jane and George.

\author{
$\Pi_{\mathrm{H}}$ : F7, F8, F15, F17, F18, F19, R1, R2, R4, R6, R7, R8, HR1. \\ $\Pi_{\mathrm{G}}: \mathrm{F} 2, \mathrm{~F} 9, \mathrm{~F} 21, \mathrm{R} 1, \mathrm{R} 7, \mathrm{R} 8, \mathrm{GR} 1$. \\ $\Pi_{\mathrm{J}}: \mathrm{F} 14, \mathrm{~F} 18, \mathrm{~F} 20 \mathrm{R} 1, \mathrm{R} 3, \mathrm{R} 5, \mathrm{JR} 1$.
}

These are sufficient to generate the dialogue given in Section 2.1.

(1) Harry can derive acceptableRestaurant (thai Palace) from F15 and HR1.

(2) George now states F2. At this point, it is unclear from his utterance whether his problem is time or money. In fact, his motivation is money, and he is trying to satisfy the second condition of GR1.

(3) Harry now states the quality argument using R1, F19 and F17.

(4) George remains concerned about price and suggests that Nosh has good food using R1 and F21, hoping that someone can supply the required fact trustworthy (students) .

(5) Harry contributes R2, so Nosh can no longer satisfy GR1.

(6) Jane now establishes the quality of La Zingara with R1, F20 and F18. JR1, however, also requires that the food be healthy. With F14, R3 and R5, she knows that the food at La Zingara is healthy and so contributes this.

(7) Harry now establishes that Thai food is also healthy by adding R4 and R6 to the already presented F15. Jane can now derive that the Thai Palace is acceptable using this information together with JR1.

(8) Having got Jane on side, Harry now turns to George. Assuming that it is cost that concerns George, he uses F7 and F8 to point out that the Thai Palace is cheaper.

(9) George remains unconvinced because of total cost. He restates F2, but giving the cost of the taxi.

(10) Harry can now say that the total cost is the same, and so GR1 is now satisfied for the Thai Palace.

What is crucial in making this dialogue a persuasion dialogue is the presence of the individual rules HR1, GR1 and JR1. During the course of the dialogue, the participants come to know things that they did not know before, both by being told and by being given information that allows them to deduce new information. For example, Harry learns that the total cost of the Thai Palace is $\$ 25$, when he is informed of the taxi fare. But these are not matters of persuasion, but rather of information sharing. Persuasion is necessary because the participants, quite properly, have their own idea of what makes a restaurant acceptable, and it is their own personal criteria that must be satisfied if they are to be persuaded. During the dialogue, participants commit to criteria representing their "point of view" or position. These differences in criteria are the source of the conflict that necessitates persuasion and, for persuasion to be successful, the proponent must demonstrate that his option satisfies the criteria to which the opponent has committed in the dialogue. Moreover, it is this that drives the utterances: when George and Jane contribute facts or rules, their contributions are guided by their personal criteria. Thus in step 2, George contributes $\mathrm{F} 2$ because he is interested in discovering the total cost of the various options. When Jane mentions the alternative in step 6, it is because La Zingara does satisfy her criteria, and she needs to be convinced that the Thai Palace also satisfies them before she can be persuaded that it is acceptable. For this reason, she invites Harry to demonstrate that the Thai Palace food is also healthy. 


\subsection{Features of a persuasion dialogue}

We can now identify some features of a persuasion dialogue (considering persuasion involving preferences rather than concerning matters of facts, when the dialogue is correcting misconceptions or completing information, rather than persuading ${ }^{4}$ ):

- Each participant has some rule that allows him or her to derive the required conclusion. These rules are local to the participants and represent the individual preferences of the participants.

- The proponent, $P$, should be able to derive the conclusion $c(a)$ at the outset. The opponent, $O$, should not be able to derive $c(a)$, as this would make persuasion unnecessary.

- $O$ may or may not be able to derive $c(b)$ for some $b \neq a$.

- An utterance by $O$ is relevant if it solicits information that would contribute to the derivation of $c(a)$ from $\Pi_{O}$, that is, information that shows that some condition of the subjective rule is satisfied. ${ }^{5}$

- $O$ should concede the point if $c(a)$ is derivable from $K B_{O}$, together with information $O$ has committed to in the course of the dialogue, irrespective of whether there is some $b \neq a$ for which $c(b)$ is derivable.

We may further note that information may be solicited by a direct question as to whether $a$ satisfies one of the conditions of the rule or by a claim that $a$ does not satisfy one of the conditions of the rule or by a statement that some $b \neq a$ satisfies the condition. In turn, $P$ should provide objective information to show that $a$ does satisfy the condition demanded by $O$.

\subsection{Features of a deliberation dialogue}

We now turn to deliberation. The first point to note is that at the outset of the dialogue, the claim is uninstantiated. The task of the dialogue is to find some $a$ for which $c(a) .{ }^{6}$ Now, at the outset, even though Harry, Jane and George can all find an instantiation of acceptableRestaurant (X), they do not commit to persuading the others. Instead, they make general observations as to what might be important features of restaurants, with a view to finding some agreed rule $A R 1$ which can be used to find an $a$ for which $c(a)$ can be derived. ${ }^{7}$

In the scenario:

(1) Harry states F15, indicating that the Thai Palace is an option and that the cuisine may be important. This is natural since it is the condition of HR1.

(2) George states F2, offering Nosh as an option and indicating that time and cost may be important. Cost, recall, is important to George.

(3) Jane now uses R1, F20 and F18 to establish the quality of the food to be important and that La Zingara is one option that satisfies this condition.

(4) Harry now mentions wine, indicating that this is important. This may be seen as strategic, in that Nosh does not satisfy HR1, or it may be that he simply feels that wine (X) should be a condition of $A R 1$, and that all three will accept this. (See also the discussion of R11 and $\mathrm{R} 12$ below.)

(5) Jane now introduces the factor of atmosphere, in a similar way.

(6) George now contributes F10, which can be seen as accepting atmosphere (X) as a condition of $A R 1$ and that La Zingara satisfies it.

(7) Harry now says that the quality at the Thai Palace is good, accepting quality as a condition of $A R 1$, satisfied by both the Thai Palace and La Zingara.

(8) George uses the taxi cost in the third argument of F2 to indicate that cost is a consideration.

(9) Harry now adds the healthy nature of the food as a possible condition and says that it is satisfied by the Thai Palace. 
(10) Jane establishes that La Zingara also satisfies this condition.

(11) George now asserts that atmosphere (accepted in step 6) and quality (accepted in step 7) are the desired conditions of $A R 1$. He has been forced to abandon Nosh as it does not meet the quality condition of GR1. He then says that both the Thai Palace and La Zingara would satisfy this rule.

(12) Jane now adds wine as a condition: this does not affect the argument, since both options satisfy it, but is a concession to Harry who proposed the condition.

(13) Harry now uses F1 and F2 to suggest distance as a tie breaker.

(14) The cost of the taxi needed by the Thai Palace is used by George for financial reasons (he would rather spend the money on the meal than on the taxi).

(15) The distance appeals to Jane because it makes things quicker.

Thus in the course of the dialogue, the participants have constructed a rule:

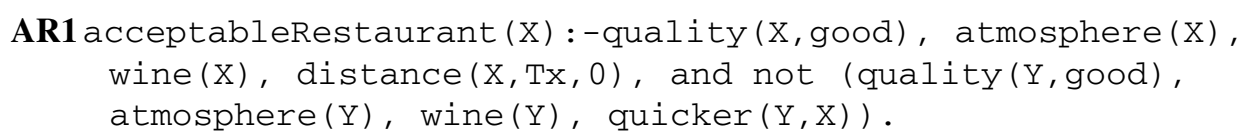

Note that the agreed rule does not contain Jane's condition relating to health, Harry's relating to cuisine or George's relating to cost. In fact, the private conditions of Jane and George are satisfied, so it seems that Harry has made a concession. In fact, however, no one endorsed his suggestion that the style of cuisine mattered, and his other suggested criteria of wine and distance were used, so he may be considered compensated. This may be particularly so if Harry also had rules in $\Pi_{H}$ such as

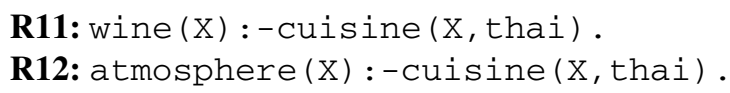

so that his HR1 implicitly includes wine and atmosphere as criteria. Equally Jane may have corresponding rules for good restaurants, if she thinks that no good restaurant would lack wine and atmosphere.

\subsection{Distinguishing the dialogues}

In the deliberation dialogue, all the participants use their private rules to inform their contributions, but they are not allowed to insist on them: it is important to establish a rule that is mutually accepted and capable of being satisfied by at least one option. Thus, we can contrast Jane's move in step 3 of the deliberation dialogue with her similar move in step 6 of the persuasion. In the persuasion dialogue, she is indicating that good quality is a condition of her private rule and that no restaurant which fails to satisfy this condition will be acceptable to her. In the deliberation dialogue, she is rather proposing quality as a condition for the agreed rule. In the three-person dialogue under discussion, conditions are brought into the agreed rule by a kind of vote: one person puts them forward and another endorses them: here Harry agrees that quality is important in step 7. In this way, the condition has the support of a majority. As well as establishing the conditions to be satisfied, the dialogue establishes which options satisfy them. Moreover, because the options are not owned in the deliberation as they are in the persuasion, and because the setting is cooperative rather than adversarial, information showing them to be satisfied can be contributed by anyone, not just by the person who introduced the option. Thus, Jane introduces atmosphere in step 5, and George shows that La Zingara satisfies it in step 7. Finally, note that the final discrimination is a new criterion, not in any of the individual preference rules: this is an example of how the preferences may be a product of the reasoning rather than an input to it (see Searle (2003) for a discussion of this point). 


\section{Contrast between persuasion and deliberation}

From the above, we remark on the following points of contrast:

(1) Persuasion starts with a conflict, whereas deliberation starts from a desire to cooperate. At the outset of a persuasion, $P$ is committed to a particular instantiation of a predicate, whereas in a deliberation, none of the participants has a commitment, but all seek to find an acceptable instantiation of the predicate. There is, therefore, no burden of proof in a deliberation dialogue, whereas in persuasion, the proponent is required to satisfy the criteria to which the opponents are committed.

(2) In a persuasion, the roles are asymmetric: we have a proponent and opponents. In a deliberation, the roles of all participants are initially the same.

(3) In a persuasion, the participants have a private decision rule, which they are entitled to see satisfied before being persuaded. In a deliberation, the aim is to satisfy some public decision rule constructed and committed to in the course of the dialogue.

(4) In a persuasion, one party is expected to supply information, the other to demand it. In a deliberation, all parties supply and request information.

The significance of the distinction between the dialogues is in the illocutionary force of various speech acts. In both types of dialogues, the participants can perform similar speech acts: they may state facts and rules, challenge facts, ask questions and so on, but what they are trying to achieve with these performatives, and what the other participants can conclude from them, is different in the two dialogue types.

Consider again the scenarios. In P1, Jane asks a question to initiate the dialogue. Note that this is the same question as is used to initiate the deliberation dialogue. At this stage, the dialogue type is not determined. In P2, Harry makes a proposal, establishing a persuasion dialogue. From this, Harry is committed to acceptableRestaurant (thai Palace). He also states a fact. The suggestion is that the style of cuisine will appear in the decision rule of George or Jane or both. In fact, this is not the case, and so this is not pursued. In P3, George states a fact. It is supposed to be understood that time or cost (or both) is a condition in his decision rule. In P4, Harry makes a claim supported by a rule and facts. Again it is to be supposed that Harry expects that quality is a condition in the decision rules of George and Jane. In P5, George makes a claim about quality. Here, he accepts that quality is a condition in his rule, but suggests that Harry's option is not the only way to satisfy this. In P6, Harry rebuts George's claim. In P7, Jane makes a claim about quality, again admitting it as a condition for her and again suggesting another option that satisfies it. She then makes another claim, indicating the second condition in her rule, satisfied by her suggestion but not, as far as she is aware, by Harry's proposal. In P8, Harry supplies information that shows Jane that her second condition is satisfied by his proposal. Since this is her last condition, Jane is now persuaded. Note that she is not committed to argue for her suggestion and should accept any option that satisfies her criteria. In P9, Harry turns to addressing George's criteria. In P10, George states a fact which shows that cost is one of his decision criteria and indicates it to be putatively not satisfied by Harry's proposal. In P11, Harry can assure George that it is satisfied, and so in P12 and P13, the dialogue is closed by first George and then Jane accepting the proposal.

The dialogues will be conducted using a set of speech acts or dialogue moves. We will use the set of speech acts given in Prakken (2006), which, although proposed for persuasion, will also be adequate for deliberation:

- $\operatorname{propose}(\mathbf{c}(\mathbf{a}))$.

- $\operatorname{claim}(\mathbf{p}(\mathbf{a}), \mathbf{J}) . J$ is a justification for $p(a)$.

- question(p(a)). 
- $\operatorname{accept}(\mathbf{c}(\mathbf{a}))$.

- The dialogue successfully terminates when all opponents have made an $\operatorname{accept}(c(a))$ move.

We will specify these speech acts in terms of the pre- and post-conditions associated with them. The pre- and post-conditions will be on the knowledge bases of the agents making the utterance and the dialogue so far. The state of the dialogue is recorded in two ways: we may make use of the dialogue history, $H$, which is the sequence of moves made so far, and a set of commitment stores, one for each agent, so that $\mathrm{Com}_{a g_{i}}$ represents the statements to which $a g_{i}$ has become committed in the course of the dialogue.

The pre- and post-conditions will represent the correct use of the moves. Thus, for example, to state that something is true in these dialogues, an agent should be able to demonstrate it from its knowledge base, and so $\operatorname{demo}\left(\Pi_{a g_{i}}, l\right)$ is a pre-condition for Claim $\left(a g_{i}, l\right)$. Of course an agent may lie, in which case the speech act is made without the pre-condition being satisfied. This is, however, a misuse of the speech act, with the misuse being reflected in the violation of the pre-condition. Agents may choose to misuse performatives strategically to mislead their opponents and so secure an advantage, but we will not discuss this further here.

It is important to also note that the pre- and post-conditions associated with a speech act are in part dependent on the dialogue type that is in progress (Barbuceanu and Fox 1996; BenchCapon 1998). This is why it is important to distinguish between the various dialogue types: if the participating agents do not share a conception of the dialogue type, they may apply different pre- and post-conditions, leading to misunderstandings and breakdown of the dialogue. Such misunderstandings may even be dishonestly exploited by an agent. Several well-known fallacies can be explained in terms of such misuse.

Finally, we draw attention to Grice's notion of conversational implicature. Grice (1975) advanced four maxims intended to capture the pragmatics of utterances in cooperative dialogues. The maxims express the cooperation principle:

Make your conversational contribution such as is required, at the stage at which it occurs, by the accepted purpose or direction of the talk exchange in which you are engaged,

which arguably must be observed if misunderstandings and conversational breakdown are to be avoided. The four maxims relate to quality (statements must be believed on adequate evidence), quantity (contributions should be as informative as the situation requires, and no more), relevance (which may be dependent on the dialogue type and its state) and manner (contributions should be clear and unambiguous). Of these maxims, relevance will be of particular concern to us here and will be the source of the special pre- and post-conditions we propose to differentiate persuasion and deliberation.

\section{Pre- and post-conditions for the speech acts}

Each of the speech acts will have a core version where the pre- and post-conditions will be a minimal set common to all proper uses of the act. We will give these first and then consider what additional conditions are imposed by their use in persuasion and deliberation. We will assume the following:

- A set $A g$ of agents: the participants in the dialogue.

- A set $L$ of locutions; the various statements, such as facts and rules, that may be made by the agents.

- For each agent, a commitment store, $\mathrm{Com}_{a g_{i}}$. These commitment stores are initially empty, but are built up over the course of the dialogue (where $\Rightarrow$ denotes the transformation of the commitment store). 
We will also include the agent making the move as an argument, additional to the locution forming the content of the speech act.

Claim $\left(\mathbf{a g}_{i}, \mathbf{l}\right)$

Claim is used to assert that something is true and, as such, it should be capable of being demonstrated by the agent using it from its knowledge base, augmented if needed by its current commitments. Also, the claim should not appear in any commitment store, since then the agent can use concede to commit to it. This gives the pre-conditions

- $\operatorname{demo}\left(\Pi_{a g_{i}} \cup \operatorname{Com}_{a g_{i}}, l\right)$

- $\neg l \in \operatorname{Com}_{a g_{j}}$ for any $a g_{j} \in A g$.

The post-condition is that the agent making the claim is committed to the claim:

- $\operatorname{Com}_{a g_{i}} \Rightarrow \operatorname{Com}_{a g_{i}} \cup l$.

\section{Why $\left(\mathbf{a g}_{i}, \mathbf{l}\right)$}

Why is used to ask for a reason for a claim. For sincerity, an agent must not believe (or be committed to) $l$, and at least one other agent must be committed to $l$. This gives the pre-conditions

- $\operatorname{not} \operatorname{demo}\left(\Pi_{a g_{i}} \cup \operatorname{Com}_{a g_{i}}, l\right)$

- for some agent $a g_{j} \neq a g_{i}, l \in \operatorname{Com}_{a g_{j}}$.

What are the post-conditions of this move? Certainly, there is a suggestion that one of the agents committed to $l$ should supply a reason, but this is dependent on the protocol being used rather than on the semantics of the speech act, and so there are no post-conditions relating to the commitment stores.

Concede $\left(\mathbf{a g}_{i}, \mathbf{l}\right)$

Concede, which in our cooperative context might be better termed accept, is used by an agent to accept a claim already made by some other agent. This may be because the agent can demonstrate it, but this is not essential: the agent needs only to be prepared for it to be taken as true for the purposes of the dialogue. However, the agent should not be committed to its negation. The pre-conditions therefore are

- for some agent $a g_{j} \neq a g_{i}, l \in \operatorname{Com}_{a g_{j}}$

- $\operatorname{not}\left(\neg l \in \operatorname{Com}_{a g_{i}}\right)$.

The post-condition is that $l$ is added to $\mathrm{Com}_{a g_{i}}$ :

- $\operatorname{Com}_{a g_{i}} \Rightarrow \operatorname{Com}_{a g_{i}} \cup l$.

\section{$\operatorname{Retract}\left(\operatorname{ag}_{i}, \mathbf{l}\right)$}

Retract is used to escape from a current commitment. The pre-condition is that $l$ must be a current commitment. Also the agent should not be able to demonstrate $l$ from its knowledge base and remaining commitments, as that would violate the maxims of cooperative dialogue:

- $\operatorname{not} \operatorname{demo}\left(\Pi_{a g_{i}} \cup \operatorname{Com}_{a g_{i}} \backslash l, l\right)$

- $l \in \operatorname{Com}_{a g_{i}}$.

The post-condition is that $l$ is removed from the commitment store of the agent:

- $\operatorname{Com}_{a g_{i}} \Rightarrow \operatorname{Com}_{a g_{i}} \backslash l$.

Since $\left(\mathbf{a g}_{i}, \mathbf{l}, \Pi\right)$ 
Since is used to reply to a why, offering a reason for a claim. The agent $a g_{i}$ must be committed to $l$, and the justification must be such as to commit the questioning agent $a g_{j}$ to $l$. The pre-conditions are

- $l \in \operatorname{Com}_{a g_{i}}$

- $\operatorname{demo}\left(\Pi \cup \operatorname{Com}_{a g_{j}}, l\right)$ for some $\Pi \subseteq \Pi_{a g_{i}}$.

The post-conditions are that both agents are committed to $\Pi$ :

- $\operatorname{Com}_{a g_{i}} \Rightarrow \operatorname{Com}_{a g_{i}} \cup \Pi$

- $\operatorname{Com}_{a g_{j}} \Rightarrow \operatorname{Com}_{a g_{j}} \cup \Pi$.

Note that here the agent providing the explanation, $a g_{i}$, may be using facts or rules present in $\mathrm{Com}_{a g_{j}}$ which it is not in fact committed to. The real reason for the agent $a g_{i}$ accepting $l$ may be quite different, but in order to persuade, it is important to give a reason that $a g_{j}$ will accept. Using such information commits $a g_{i}$ to using it for the purposes of the dialogue, but need have no effect on $\Pi_{i}$. Thus, an agent may itself prefer a restaurant because it is cheap, but will give a lover of Thai food the reason that it serves Thai food.

\section{Question $\left(\mathbf{a g}_{i}, \mathbf{l}\right)$}

Question is used to seek information. This means that no agent should be currently committed to $l$ (otherwise why should be used), nor should the asking agent believe $l$ (when claim should be used). The pre-conditions are thus

- $\forall\left(a g_{j}\right) \in A g, l \notin \operatorname{Com}_{a g_{j}}$

- $\operatorname{not} \operatorname{demo}\left(\Pi_{a g_{i}} \cup \operatorname{Com}_{a g_{i}}, l\right)$.

Again there are no universal post-conditions, although particular protocols may require an agent to respond to the question with a claim of $l$ or one of its contraries. There will, however, be post-conditions associated with the particular dialogue types. We will therefore now look at the particular dialogues, starting with persuasion.

\subsection{Persuasion}

Persuasion arises from disagreement: at least one participant believes that a particular option is acceptable and one or more others do not. How can such a disagreement arise? Some disagreements are inevitable: since agents may look for different criteria, it will often be the case that an option acceptable to one agent will not be acceptable to another. In such a case, persuasion should fail. But as well as these differences in preferences, there will also be differences in information. One agent may well not recognise the best available option through ignorance of some facts about the various options, so that an option may in fact best satisfy its criteria without it being aware that this is so. Note, however, that it is the criteria of the agent being persuaded that matter: the reasons that attract the persuader to the option may differ. Recall the persuasion scenario presented above, in Section 2.

During the course of that dialogue, Jane and George reveal their individual criteria. In P3, George's claim that the Thai Palace is far away is made because cost is one of his criteria in GR1, and his claim that Nosh is of good quality in P5 indicates the other condition in GR1. Similarly, Jane reveals the two criteria in JR1 in P7. Thus, in order to be relevant, claims made by $O^{8}$ must be required to establish the conclusion using the individual preference rule of $O .{ }^{9}$ Similarly, if $O$ seeks a reason for one of $P$ 's claims, $O$ is accepting that that claim is relevant to the satisfaction of $O$ 's individual preference rule. The key point here is that several of the moves have, in this context, implications that go beyond the basic conditions described in the previous section. Also 
these pragmatic implications differ according to the role of the speaker. Therefore, the opponent will only make claims relating to features that may lead him to prefer a particular option. So, any claim will correspond to a feature (say $p$ ) in the body of $O$ 's preference rule.

Thus, we have the additional pre-conditions for $O$ to make the move $\operatorname{claim}(O, p(a))$ :

- $\operatorname{demo}\left(\Pi_{O} \cup \operatorname{Com}_{O}\right.$, acceptableRestaurant $\left.(a)\right)$

- $p(X)^{10} \in B$, where $B$ is the set of terms in the body of the preference rule of $O$.

Similarly, the post-conditions now also include this information:

- $\operatorname{Com}_{O} \Rightarrow$ Com $_{O} \cup$ acceptableRestaurant(a)

- $\operatorname{Com}_{O} \Rightarrow \operatorname{Com}_{O} \cup(p(X) \in B)$, where $B$ is the set of terms in the body of the preference rule of $O$.

The situation is the same when $O$ poses questions. Again, $O$ should ask only about criteria which he in fact finds desirable. Thus, question $(O, p(a))$ also gets the additional pre-conditions:

- $\operatorname{not}\left(\operatorname{demo}\left(\Pi_{O} \cup \operatorname{Com}_{O}, p(a)\right)\right.$

- $p(X) \in B$, where $B$ is the set of terms in the body of the preference rule of $O$,

and this commitment is recorded with the additional post-condition

- $\operatorname{Com}_{O} \Rightarrow \operatorname{Com}_{O} \cup(p(X) \in B)$, where $B$ is the set of terms in the body of the preference rule of $O$.

The agent doing the persuasion, $P$, can make claims about the attributes of the favoured option, but these will only be relevant to $O$ if they form part of the body of $O$ 's preference rule. If the attribute is not a criterion for $O$, it can be simply ignored. If, however, it is an attribute that forms part of the body of $O$ 's preference rule and so matters to $O$, then $O$ should accept the claim with concede. In doing so, $O$ becomes committed to this criterion. Thus, concede $(O, p(a))$, like question $(O, p(a))$, has the additional pre-condition

- $p(X) \in B$, where $B$ is the set of terms in the body of the preference rule of $O$,

and post-condition

- $\operatorname{Com}_{O} \Rightarrow \operatorname{Com}_{O} \cup(p(X) \in B)$, where $B$ is the set of terms in the body of the preference rule of $O$.

Two key points emerge here. One is the asymmetry present in the dialogue. This reflects the asymmetry of the treatment of the criteria in the bodies of the agents' individual preference rules: it is the criteria held by $O$ that are important, for it is these that must be shown to be satisfied in order for $O$ to be persuaded. Persuasion arises out of incomplete information, and so $P$ is trying to supply information that $O$ does not have but which would satisfy its criteria. Similarly, $O$ is interested in indicating the criteria it requires information about. Thus, $O$ can use claim, question and concede to indicate the criteria in which it is interested, because they will satisfy its preference rule. In contrast, $P$ has no need to indicate its own criteria, but should avoid supplying unwanted information relating to features in which $O$ is not interested. The purpose of the pragmatic implicature of the moves in a persuasion dialogue is to maintain relevance by informing $P$ of $O$ 's preference rule, so that $P$ can be aware of the facts that $O$ needs to adopt the desired preference.

In the next sub-section, we look at the additional conditions that apply to deliberation. 


\subsection{Deliberation}

We now consider deliberation using the second scenario given in Section 2.2. In this dialogue, the participants begin by making claims about certain features of restaurants. When they make these claims, they do not in fact care whether the facts are already known to the other agents. This might seem redundant, but these claims are not primarily intended to impart information, although they do have this effect for agents who happen to be unaware of the information. These claims are meant to indicate criteria that the speaker would be happy to be included in the group criteria, that is, to appear in the body of the agreed preference rule that the deliberation dialogue is intended to construct. Claim therefore shares the pre- and post-conditions of claim as used by $O$ in a persuasion dialogue. We can thus see moves like D2-D4 as effectively proposing criteria, as well as sharing awareness of the attributes of the various restaurants. As with persuasion, criteria can also be introduced through a question move, if the participant wants to propose an attribute for inclusion in the decision rule, but does not know of an option satisfying it.

In D13, George proposes a concrete rule satisfying some of the criteria previously advanced in the dialogue. Here, George is using the since move and so making claims about the criteria which are, or should be, acceptable to the group. Similarly, in D15, Harry uses a since move to add an extra, discriminating, criterion to the agreed rule.

The key move here is since. Since makes a commitment to the group criteria found in the body of the agreed preference rule $A R 1$. In order to propose that a criterion be adopted as part of the agreed rule, it must previously have been made into a candidate by being introduced to the discussion. Moreover, this introduction should have been by some other agent, since otherwise there would be no reason to suppose general support for this criterion. The additional pre-conditions for since $\left(a g_{i}, g(a), p(a)\right.$ ) (where $g(a)$ is the goal of the deliberation, e.g. in the scenario acceptableRestaurant $(a))$ in deliberation are therefore

- $\operatorname{demo}\left(\Pi_{a g_{i}} \cup \operatorname{Com}_{a g_{i}} \cup p(a), g(a)\right)$

- $p(a) \in \operatorname{Com}_{a g_{j}}$ for some $a g_{j} \neq a g_{i}$.

This reflects that the option should be acceptable to the agent providing the reason, but the criterion should have previously been committed to by some other agent. There is also the additional post-condition

- $\operatorname{Com}_{O} \Rightarrow \operatorname{Com}_{O} \cup p(X) \in B$, where $B$ is the set of terms in the body of the preference rule $A R 1$.

Similarly, if the agent uses concede, it recognises that the conceded attribute should be part of the agreed rule. That the conceded fact is already in some other agent's commitment store and not in that of the conceding agent is already part of the general pre-conditions. In deliberation, however, there is an additional post-condition. Thus, concede $\left(a g_{i}, p(a)\right)$ has the additional post-condition

- $\operatorname{Com}_{O} \Rightarrow \operatorname{Com}_{O} \cup p(X) \in B$, where $B$ is the set of terms in the body of the preference rule $A R 1$.

\section{Discussion}

We are now in a position to characterise the essential differences between persuasion and deliberation. The differences arise from the criteria that will be used to justify the choice at the conclusion of the dialogue. In persuasion, these criteria are determined by only some of the participants, the agents being persuaded, on the basis of individual preferences. If several agents need to be persuaded, they can, and typically will, use different criteria since their preferences will typically vary. In contrast, at the start of a deliberation dialogue, the criteria are not yet fixed and need to 
be formed from a selection of the criteria subscribed to by the participants during the course of the dialogue. In deliberation, only one set of criteria, representing the preference of the group as a whole, will be used. Thus in persuasion, the personal criteria of the agents being persuaded need to be elicited so that the persuader can show that the option satisfies them. In deliberation, the criteria of all the participants need to be introduced and then chosen from to form a decision rule acceptable to the group collectively.

As we saw in the previous section, the various speech acts take on additional pre- and postconditions depending on the context provided by the dialogue type. These additional pre- and post-conditions represent the pragmatic forces of the speech acts, derived from their conversational implicatures in terms of relevance (Grice 1975; Walton 2004), which is in turn determined by their relationship to the criteria for acceptability being revealed (persuasion) or constructed (deliberation).

In both persuasion and deliberation, the motivation for the agents to make claims and ask questions is first and foremost to reveal the criteria used in their individual preference rules, which the agents can then attempt to satisfy. This is what keeps the dialogue focused on what must be established if the dialogue is to reach a successful conclusion. In making claims, there is no need for the participant to believe that the others do not know what is being claimed. For example, when it is claimed that the Thai Palace serves Thai food in a persuasion dialogue, the point of the utterance is to communicate the information that the speaker regards Thai cuisine as a reason to go to that restaurant. In contrast, in a deliberation dialogue, the point will be that this is an attribute that the agent would be happy to see form part of the collective decision rule. The additional requirement of deliberation dialogues, to enable criteria to move from mere candidates favoured by some agent to those to be applied by the group, is accomplished by the justifying move since, which explicitly states a reason in favour of an option, and, in doing so, implicitly commits the speaker to that reason being a reason that the group should use. Similarly, concede can be used to approve a candidate criterion forming part of the collective rule. In order to understand what is happening in these dialogues therefore, it is vital to recognise that they are providing the context for the pragmatic meanings of the various utterances indicated above.

\subsection{Discussing matters of fact}

If we consider persuasion as described in Prakken (2006), persuasion takes place in the following ways:

- One participant supplies the other with some fact unknown to that other participant, which enables the claim to be deduced.

- One participant supplies the other with some rule unknown to that participant, which enables the claim to be deduced.

- An inconsistency in one participant's belief base is demonstrated, so that a claim, or an objection to a claim, is removed.

At least one of these must occur for persuasion about a factual matter to happen, and in a complicated persuasion dialogue, all three may be required. This requires certain further assumptions about the context: that the beliefs of the participants are individually incomplete or collectively inconsistent. That is, the beliefs of the participants are defective in some way and are corrected or completed through the dialogue. In our persuasion scenario, all three means of persuasion occur: facts about the options are established, rules allowing relevant facts to be deduced are exchanged and misconceptions are corrected (although this is done through exchanging rules, so blocking inferences previously possible using negation as failure). 
Note, however, that throughout our persuasion scenario, the agents are discussing matters of fact, rather than subjective preference, and the desired conclusion can be shown only if it can be shown from the union of the knowledge base of $O$ (which contains the required preference rule) with a subset of the knowledge base of $P$. Again persuasion exhibits an asymmetry, in that the persuader is able to conceal knowledge that would be harmful to its cause. If $O$ favours a criterion that is, unknown to $O$, satisfied by its currently preferred option, $P$ would be best advised to conceal the information needed to establish this. Note also that $P$ has an additional means of persuasion available. Recall that when $O$ makes a claim, it reveals not only a criterion, but also an acceptable option. $P$ can attempt to show that the currently preferred option does not, in fact, satisfy this criterion, thus advancing the case for its own option by making its rival less acceptable to $O$. It is of course important that some option be acceptable (even if it is the lesser of two evils), and so if $O$ 's current preference rule turns out to be unsatisfiable, another rule must be used. In practice, $O$ will have a number of different preference rules, ordered by their priority. This is further discussed in Section 6.3.

With respect to deliberation, the situation is different in so far as there is no asymmetry, and the agents should wish the knowledge of all agents to be as complete as possible. Exploring the truth or falsity of matters of fact thus becomes an inquiry dialogue rather than a persuasion. For this purpose, the inquiry dialogue of Black and Hunter (2009) would be suitable, since it effectively allows all and only those inferences that can be produced from the union of the knowledge bases of the agents concerned.

\subsection{Shifts between deliberation and persuasion}

In our scenarios given in Section 2, we have tried to stay within one dialogue type. Often, however, a dialogue will contain nested dialogues, of the same or different type, and dialogues may shift, licitly or illicitly, between types. The most usual type of dialogue to be embedded within persuasion and deliberation will be information seeking. In both persuasion and deliberation, facts need to be established, and if these are unknown to the participants, the information may need to be sought elsewhere. For example, if no one knew whether Thai cuisine was healthy or not, and Harry was unwilling to concede defeat in the persuasion scenario, he could initiate an informationseeking dialogue with an agent with the requisite dietary knowledge. Harry's move in P6, where he corrects George's misconception about the quality of Nosh, could be seen as an embedded persuasion dialogue, albeit persuasion as to a matter of fact. In a cooperative dialogue, where there is no question of the strategic suppression of information for persuasive purposes, there is little difference between information seeking and persuasion as to matters of fact. The embedding of information seeking (and persuasion about matters of fact) forms a natural and necessary part of both persuasion and deliberation, since the facts must be established before the decision is made.

More interesting are shifts from one type of dialogue to another. Sometimes, a shift may become necessary: if a deliberation fails to achieve a consensus, then a shift to persuasion or, more usually, negotiation may be required to reach a decision. Some shifts, however, particularly if illicit or unnoticed, are a source of fallacies, as is emphasised in Walton and Krabbe (1995). As an example, Walton and Krabbe describe (pp. 109-110) how argumentum ad baculum (appeal to force) can be seen as an illicit shift from a persuasion dialogue (in which threats are inappropriate) to a negotiation dialogue, where threats can have a legitimate role. What would be the consequence of a shift from persuasion to deliberation? Here, we might see $P$, recognising that his option does not in fact satisfy $O$ 's criteria, attempting to make a shift to a deliberation dialogue. If successful, $O$ can no longer decide on the basis of $O$ 's own preference rule, but rather has to use some consensual rule that may lack the unsatisfiable criteria of $O$ 's rule and perhaps instead contain some criteria from $P$ 's preference rule. If $O$ fails to notice the shift and accepts the shift to deliberation, then $O$ 
may fail to reject $P$ 's favoured option, as would be quite proper in a persuasion dialogue, where $O$ is entitled to insist that his own criteria be satisfied. Thus, shifting from persuasion to deliberation effectively removes the burden of proof from $P$ and gives both participants equal power in the decision-making, whereas $O$ has the right be the arbiter in a persuasion dialogue.

Also common is a shift from deliberation to persuasion. Here, the shift can arise in two ways. First an agent might adopt the role of persuader. Whereas in a deliberation there should be no 'ownership' of options, an agent may lose sight of this and become the champion of a particular option. There might be little harm in this (since the persuader is effectively relinquishing the power to decide to the other participants), but problems can still arise because the commitments made by the agents differ between the dialogue types. Thus, in our scenario, in D7, Jane claims that Nosh lacks atmosphere. In the context of deliberation, this commits her to no more than a willingness to see atmosphere as part of the collective rule. If, however, this had been a persuasion dialogue, she would have been committed to atmosphere as being part of her decision rule and so to rejecting any option without atmosphere. In this way, an illicit shift to persuasion could force Jane to reject an option lacking atmosphere, even though this is not required by her preference rule and even though this had not been adopted as part of the collective decision rule. So, this shift is somewhat two edged. By moving from a participant in a deliberation to the persuader in a persuasion, the agent gives up some decision-making power, but can force unintended commitments on the other participants, if they fail to notice the shift. This may have strategic advantage: moreover, a subsequent shift back to deliberation can then leave the opponents with the unwelcome commitments, but without the extra decision-making power.

A second way in which an agent may try to shift from deliberation to persuasion is through attempting to assume the role of $O$. The object here is to arrogate the decision-making power for itself. If the shift is successful, it will be able to use its own preference rule as the collective rule and foist a burden of proof on the other agents. In such a situation, the agent should be aware that in making the shift it will become committed to any criteria it has previously introduced into the dialogue.

Thus, shifts from one dialogue type to another are something that participants in a dialogue need to be aware of, and avoid, if they are to avoid having different rules from those they intended applied to their utterances and if the objectives of the dialogue are to remain constant. ${ }^{11}$ Shifts, and further examples of analyses of them, are further discussed in Walton (2009).

\subsection{Reasons for criteria}

Supplying reasons for why an agent adopted the criteria used in its preference rules, using, for example, the since move, might also be used to enrich the possibilities for persuasion. If the agent currently has a rule that it is unable to apply, perhaps because some condition is known to be satisfied, then such information might lead it to adopt a different rule. For example, if an agent explains its preference for Thai food on the grounds of its health-giving properties, other agents may also develop a preference for that cuisine. Equally an agent may abandon criteria if they are shown to be based on an incorrect inference: for example, if it discovers that French food is not so healthy after all. There is, however, an important difference between preference rules and factual rules when $P$ has a rule that $O$ does not currently recognise. Where a rule concerns a matter of fact, the agents should adopt it (we are assuming that all the participants are cooperative and sincere $^{12}$ ), but this is by no means clear when it concerns a reason for desiring something, that is, the individual preference rules. Suppose, for example, an agent suggests that having Indian cuisine is a reason for favouring a restaurant and is then asked to justify this. It might well reply that Indian cuisine should be a criterion because it is spicy. This can be a perfectly acceptable reason for one person and yet unconvincing to another whose digestion is delicate and whose taste lies towards 
different food. Thus, eliciting reasons for criteria from $O$ through use of why, and then attempting to satisfy them, is more likely to be persuasive than proposing rules that are themselves subjective. This, however, represents a level beyond what we have thus far been discussing: we are now in the realms of reasons for reasons, and this we will leave for further exploration in future work.

In deliberation also, proposal of rules for endorsing criteria may be useful in encouraging others to support their adoption by the group, but will not be decisive, since these rules can legitimately reflect the taste of a single agent. Agents may even support criteria for different reasons: one may like Italian food because it is healthy, while another simply likes the taste. Here, the subjective element means that we cannot derive the criteria of the group from the union of the knowledge bases of the group and the union of the rules for adopting criteria. Instead, the criteria for a condition being adopted as part of the collective preference rules needs to be endorsed by each agent according to its own tastes.

\subsection{Preference functions}

In the examples given above, the individual preference rules have been straightforward: the restaurant is acceptable if it satisfies some list of criteria. In practice, however, criteria will differ in importance, and so determining acceptability will be a more complicated procedure, which may involve different approaches such as voting methods, as discussed in McBurney et al. (2007), or quantitative approaches to handling preference change (Liu 2009). Relating more to our framework is the potential for the use of several rules, and weighted criteria, which we discuss below.

First, we should distinguish between essential and desirable criteria. If, for example, one has only a limited time to eat (perhaps during the lunch break of a conference), then quick service may be an essential feature of the restaurant and may lead to it being chosen no matter how less desirable than its rivals it is in other respects. The key point here is that an acceptable option must satisfy all essential criteria; desirable criteria can then be used to discriminate between those options that meet these essential criteria. The implications for persuasion are that the essential criteria must be shown to be met or shown not to be essential. If quick service is said to be essential and an agent is attempting to persuade to an option that does not have this feature, the reason must be shown to be inapplicable or at least not essential. For example, if quick service is posited as essential because the conference restarts in an hour, it has to be argued that the rule either is not satisfied (e.g. because the lunch break is in fact two hours) or need not be applied (e.g. because the after-lunch session is of no interest). If such moves are successful, the criterion is no longer essential: it may be abandoned altogether ( $o k$, so we can take as long as we like) or retained as a desirable criterion ( $o k$, but I still like quick service). Some mechanism for making this distinction needs to be provided. In our simple treatment, all criteria are essential. If, however, we added another rule, say GR2, to be evaluated before the current GR1, with an additional criterion in the body, this would represent a desirable attribute. We could add several rules in this way, indicating different combinations of desirable criteria, with their degree of desirability being indicated (in our Prolog programs) by the order in which they occur. There must always, however, be at least one rule, and this will contain the essential criteria, and all the other rules must include these criteria in their bodies.

In deliberation, a similar distinction between essential and desirable attributes can be drawn and several rules proposed. Here, however, if even a single participant can claim (and defend the claim) that a criterion is essential for him, then that criterion has to be adopted as essential by the group. If only one member of the group has to catch a train, then it is essential to go somewhere with quick service or abandon the notion of eating together.

If we allow weights on criteria, then the acceptability of an option may turn not on whether an option satisfies a criterion or not, but on which of the two criteria is preferred. Thus, it may 
be accepted that two restaurants are acceptable in all respects, except that one is close by and the other has quick service. Now persuasion may be a matter of inducing the persuadee to change his preference order from closeby $\prec$ quickService to quickService $\prec$ closeby. This in turn suggests other rules, the conclusions of which will be such preference orderings. For example, we could argue that if taxis are readily available, then closeby $\prec$ quickService. Like reasons for including and excluding criteria, however, such rules are unlikely to be coercive: an agent is always free to reject them. This then is a matter for genuine persuasion rather than inquiry. ${ }^{13}$

Less clear cut is what happens to preference orders in deliberation. Each participating agent will have its own preference order: how can these be combined to give a group order? Of course, arguments can be presented as above to attempt to align them, but there is no guarantee that such arguments will succeed. It is possible to merge the orderings into a partial order consistent with all the individual orders, but this may be insufficient to resolve issues. This then may prove a problem for deliberation, especially if too many criteria are included in those accepted by the group. The question of reaching an agreement on numeric weights rather than an ordering is, we would suggest, too difficult to contemplate, which is why numeric weights do not in practice feature in such discussions. Some discussion of these issues can be found in Bench-Capon and Prakken (2010) and Bench-Capon, Prakken, and Visser (2011), but since our main purpose in this paper is to characterise the different kinds of dialogues rather than explore the nature of preference functions, the single rule expressing essential conditions is adequate for our current purposes.

\section{Conclusion}

In this paper, we have drawn a clear distinction between persuasion and deliberation dialogues in cases where the individual preferences of the participants are important. We have done so in terms of additional pre- and post-conditions that apply to the common set of speech acts depending on the dialogue type being used. These additional conditions are motivated by the different conversational implicatures appropriate to the different types, which we observe from consideration of detailed scenarios. Where there are no individual preferences, persuasion becomes more akin to the inquiry dialogues of Black and Hunter (2009). In our deliberation, we have considered a cooperative situation in which a consensus is sought. If the assumption of cooperation is relaxed, we enter rather a negotiation dialogue, in which concessions are used to effect a compromise. We leave the detailed exploration of the relation between deliberation and negotiation for future work.

\section{Notes}

1. http://debatepedia.idebate.org/en/index.php/Past_Debate_Digest_topics (accessed November 18, 2011).

2. It is possible to distinguish between different subtypes of persuasion and deliberation. For example, Prakken (2006), following Walton and Krabbe (1995), identifies a subtype of persuasion which it calls dispute, where the participants have opposed views and are engaged in mutual persuasion, and also distinguishes between permissive and rigorous dialogues. Because of the importance we attach to the pragmatic aspects, we need quite specific examples and so will focus on the specific, but representative, dialogues in our scenarios. The general point about the conversational implications, and their importance, will be true of other subtypes, but they may well have some of their own specific contextual pre- and post-conditions, which will need to be discovered by analysing examples of these other dialogue subtypes.

3. For ease of reference, we label facts beginning with F1 and rules beginning with R1.

4. We will discuss persuasion as to matters of fact further in Section 6.1.

5. This consequence of Grice's (1975) maxims (further discussed in Section 4) is also the basis of relevance in Walton (2004), which provides criteria for determining relevance in accordance with dialogue profiles Krabbe (1999). 
6. Note that whereas in persuasion, there is a specific option $a$ to which the persuader is committed, in deliberation, an option must be found. Technically, the query for persuasion involves the ground literal, whereas in the query for deliberation, the variable is free.

7. As the dialogue unwinds, conditions for the body of $A R 1$ will be proposed and adopted, so that it changes during the dialogue. We will state only the final form of $A R 1$ at the end of the dialogue.

8. We will continue to use $P$ and $O$. In persuasion, these stand for proponent and opponent, but in the cooperative context of deliberation, they refer to proposer and other.

9. Here, we assume that $O$ has a single preference rule, although in practice this rule might contain a disjunction in the body and so correspond to several Prolog rules, where the order of the rules will prioritise the disjuncts.

10. Although we have $p(a)$ in the locution, the rules will have the variable of $p$ uninstantiated, since they are intended to apply to any option with this feature.

11. It is also possible that the agents may simply differ as to the type of dialogue they are engaged in. This presents serious dangers of misunderstanding. Ideally, in a formal setting, the protocol should make it clear what type of dialogue is being entered into, so that there is no ambiguity. In informal settings, the context in which the dialogue starts should determine the type of dialogue.

12. Sincerity is a strong condition, and it is a more plausible assumption for deliberation than for persuasion, where often the agents are self-interested. In our scenarios, the persuader is expected to have the persuadee's best interests at heart and so should be sincere. A form of insincerity in persuasion and its effects on conversational implicature are considered in Atkinson, Bench-Capon, and Bench-Capon (2012), but here we give conditions for sincere use of the performatives and expect agents to adopt strategies to cope with violations of these conditions outside the protocol.

13. Computational machinery for arguing about preferences in this way is given in Modgil (2009).

\section{References}

Amgoud, L., Maudet, N., and Parsons, S. (2002), 'An Argumentation-Based Semantics for Agent Communication Languages', in Proceedings of the 15th European Conference on Artificial Intelligence, ECAI'2002, Lyon, France, July 2002. ed. F. van Harmelen IOS Press, Amsterdam, pp. 38-42.

Atkinson, K., Bench-Capon, P., and Bench-Capon, T. (2012), 'Efficiency in Persuasion Dialogues', in Proceedings of the Fourth International Conference on Agents and Artificial Intelligence (ICAART 2012), Vol. 2 - Agents, Vilamoura, Algarve, Portugal, 6-8 February, 2012, SciTe Press, pp. 225-230.

Barbuceanu, M., and Fox, M.S. (1996), 'The Design of a Coordination Language for Multi-Agent Systems', in ATAL, eds. J.P. Müller, M. Wooldridge, and N.R. Jennings, Vol. 1193 of Lecture Notes in Computer Science, Berlin: Springer, pp. 341-355.

Bench-Capon, T.J.M. (1998), 'Specifying the Interaction Between Information Sources', in DEXA, eds. G. Quirchmayr, E. Schweighofer, and T.J.M. Bench-Capon, Vol. 1460 of Lecture Notes in Computer Science, Berlin: Springer, pp. 425-434.

Bench-Capon, T. (2002), 'Agreeing to Differ: Modelling Persuasive Dialogue Between Parties Without a Consensus About Values', Informal Logic, 22, 231-245.

Bench-Capon, T.J.M., and Prakken, H. (2010), 'A Lightweight Formal Model of Two-Phase Democratic Deliberation', in JURIX 2010: The Twenty-Third Annual Conference on Legal Knowledge and Information Systems, Liverpool, UK, 16-17 December 2010. ed. R. Winkels, Vol. 223 in Frontiers in Artificial Intelligence and Applications, IOS Press, Amsterdam, pp. 27-36.

Bench-Capon, T.J.M., Prakken, H., and Visser, W. (2011), 'Argument Schemes for Two-Phase Democratic Deliberation', in The 13th International Conference on Artificial Intelligence and Law, eds. Kevin D. Ashley, and Tom M. van Engers, June 6-10, PA, USA. ACM Press 2011, New York. pp. 21-30.

Black, E., and Atkinson, K. (2009), 'Dialogues that Account for Different Perspectives in Collaborative Argumentation', in 8th Int. Joint Conf. on Autonomous Agents and Multi-Agent Systems, Budapest, Hungary, May 10-15, 2009, Volume 2. IFAAMAS, SC, pp. 867-874.

Black, E., and Hunter, A. (2009), 'An Inquiry Dialogue System', JAAMAS, 19, 173-209.

Dignum, F., Dunin-Keplicz, B., and Verbrugge, R. (2001), 'Creating Collective Intention Through Dialogue', Logic Journal of the IGPL, 9, 289-304. 
Dunin-Keplicz, B., Strachocka, A., and Verbrugge, R. (2011), 'Modeling Deliberation in Teamwork', in AAAI Spring Symposium: Logical Formalizations of Commonsense Reasoning, Papers from the 2011 AAAI Spring Symposium, Technical Report SS-11-06, Stanford, California, USA, March 21-23, 2011. AAAI, CA.

Grice, H. (1975), 'Logic and Conversation', in Syntax and Semantics, Vol. 3, eds. P. Cole and J. Morgan, New York: Academic Press, pp. 43-58.

Krabbe, E. (1999), 'Profiles of Dialogue', in JFAK: Essays Dedicated to Johan van Benthem on the Occasion of his 50th Birthday eds. J. Gerbrandy, M. Marx, M. de Rijke, and Y. Venema, Amsterdam: Amsterdam University Press, pp. 25-36.

Liu, F. (2009), 'Preference Change: A Quantitative Approach', Studies in Logic, 2, 12-27.

McBurney, P., Parsons, S., and Wooldridge, M. (2002), 'Desiderata for Agent Argumentation Protocols', in Proceedings of the First International Joint Conference on Autonomous Agents and Multiagent Systems (AAMAS ’02), Bologna, Italy, July 15-19, 2002, New York, NY: ACM, pp. 402-409.

McBurney, P., van Eijk, R.M., Parsons, S., and Amgoud, L. (2003), 'A Dialogue Game Protocol for Agent Purchase Negotiations', Autonomous Agents and Multi-Agent Systems, 7, 235-273.

McBurney, P., Hitchcock, D., and Parsons, S. (2007), 'The Eightfold Way of Deliberation Dialogue', International Journal of Intelligent Systems, 22, 95-132.

Modgil, S. (2009), 'Reasoning About Preferences in Argumentation Frameworks', Artificial Intelligence, $173,901-934$.

Prakken, H. (2006), 'Formal Systems for Persuasion Dialogue', Knowledge Engineering Review, 21, $163-188$.

Rahwan, I., Ramchurn, S.D., Jennings, N.R., McBurney, P., Parsons, S., and Sonenberg, L. (2003), 'Argumentation-Based Negotiation', KER, 18, 343-375.

Reed, C. (1998), 'Dialogue Frames in Agent Communication', in ICMAS, 3-7 July 1998, Paris, France, ed. Y. Demazeau, IEEE Computer Society, pp. 246-253.

Reed, C., and Norman, T. (eds.) (2003), Argumentation Machines: New Frontiers in Argumentation and Computation, Dordrecht: Kluwer.

Searle, J.R. (2003), Rationality in Action, Cambridge, MA: The MIT Press.

Tang, Y., and Parsons, S. (2005), 'Argumentation-Based Dialogues for Deliberation', in 4th Int. Joint Conf. on Autonomous Agents and Multi-Agent Systems, July 25-29, 2005, Utrecht, The Netherlands. ACM, NY. pp. 552-559.

Walton, D. (1998), The New Dialectic, Toronto: University of Toronto Press.

Walton, D. (2004), Relevance in Argumentation, Mahwah, NJ.: Lawrence Erlbaum Associates.

Walton, D. (2006), Fundamentals of Critical Argumentation, Cambridge, MA: Cambridge University Press.

Walton, D. (2009), 'Dialectical Shifts Underlying Arguments from Consequences', Informal Logic, 29, $54-83$.

Walton, D., and Krabbe, E. (1995), Commitment in Dialogue: Basic Concepts of Interpersonal Reasoning, Albany NY: State University of New York Press. 